\title{
The concept and controversy of retroperitoneal nerve dissection in pancreatic head carcinoma (Review)
}

\author{
XUAN WANG $^{1}$, HONGWEI ZHANG ${ }^{1}$, TAIHONG WANG ${ }^{1}$, WAN YEE LAU ${ }^{2}$, \\ $\mathrm{XIN}_{\text {WANG }}{ }^{3}$, JINGFENG SUN ${ }^{1}$, ZHENHUA YUAN $^{1}$ and YEWEI ZHANG ${ }^{1}$ \\ ${ }^{1}$ Department of Hepatobiliary and Pancreatic Surgery, Affiliated Jiangsu Cancer Hospital of Nanjing Medical University, \\ Nanjing, Jiangsu 210009; ${ }^{2}$ Department of Surgery, The Chinese University of Hong Kong, Prince of Wales Hospital, \\ Shatin, Hong Kong SAR; ${ }^{3}$ Department of Oncology, Affiliated Jiangsu Cancer Hospital \\ of Nanjing Medical University, Nanjing, Jiangsu 210009, P.R. China
}

Received July 13, 2015; Accepted September 15, 2015

DOI: $10.3892 /$ ijo.2015.3190

\begin{abstract}
Pancreatic head cancer is a common but the most lethal cancer of the human digestive system. It is invasive, resulting in early infiltration of adjacent structures and lymph node and distant metastases. Its biological characteristics of neurotropic growth lead to early neural invasion (NI) which is an independent prognostic factor of survival for pancreatic cancer. Radical surgical resection remains the only form of curative treatment. The extent of surgical resection and whether extended resection results in better long-term survival have been controversial. Studies have reported that peripancreatic plexus invasion is a frequent cause of pancreatic cancer recurrence and death. The relationship between cancer microenvironment and nerve cells, and whether the peripancreatic nerve plexus nearby needs to be resected require further studies. The present review aims to discuss the role of peripancreatic nerve and its implications in pancreatic head cancer resection.
\end{abstract}

\section{Contents}

1. Introduction

2. Anatomy and factors related to neural invasion of pancreatic carcinomas

3. Factors associated with neural invasion of pancreatic carcinoma

4. Molecular biological mechanism of neural invasion in pancreatic carcinomas

Correspondence to: Dr Yewei Zhang, Department of Hepatobiliary and Pancreatic Surgery, Affiliated Jiangsu Cancer Hospital of Nanjing Medical University, Nanjing, Jiangsu 210009, P.R. China

E-mail: zhangyewei@njmu.edu.cn

Key words: pancreatic head carcinoma, retroperitoneal, mesopancreas, nerve dissection, optogenetic technology
5. Composition of pancreatic tumor microenvironment

6. Tumor-neural microenvironment

7. Surgical treatment, adjuvant therapy, and monitoring technology to follow neural invasion in pancreatic carcinoma

8. Treatment of retroperitoneal nerve dissection and vascular skeletonization

9. Optogenetic technology with pancreatic cancer for detection and adjuvant therapy

10. Conclusions

\section{Introduction}

The pancreas is a retroperitoneal organ. The peripancreatic nerves form a structure of network arranged in a crisscross pattern like clouds in the retroperitoneum, spreading along the abdominal aorta and its main branches, around the celiac artery and at the root of the superior mesenteric artery within the retroperitoneal soft tissues. In addition to the common biological behavior of cancers that come with direct invasion, hematogenous spread and lymphatic metastasis, pancreatic cancers have unique neurotropic growth characteristics which result in early perineural invasion. Some scholars (1) believe in neural invasion (NI), tumor cells grow along a nerve in any layer including the endoneurium, perineurium or epineurium. However, the exact mechanism of how NI occurs is still unclear (2). One theory proposes that NI occurs in a low resistance fissure between the pancreas and the nervous system. When tumor cells infiltrate the epineurium, immune injury by invasive tumor cells significantly alter the microenvironment making it further conducive to tumor invasion and metastasis along the neurons, eventually causing pain (3). Other studies show NI occurs because of secretion of neurotransmitters between the tumor cells and the nerves which attract tumor cells. Many signal molecules, including neurotrophic factors, cytokines, and cell surface ligands/receptors are involved in NI. Intraoperative nerve dissection methods, scope, and postoperative treatment have been the main foci in pancreatic cancer research to study the basis of pancreatic neural invasion and the mechanisms of neural invasion. 

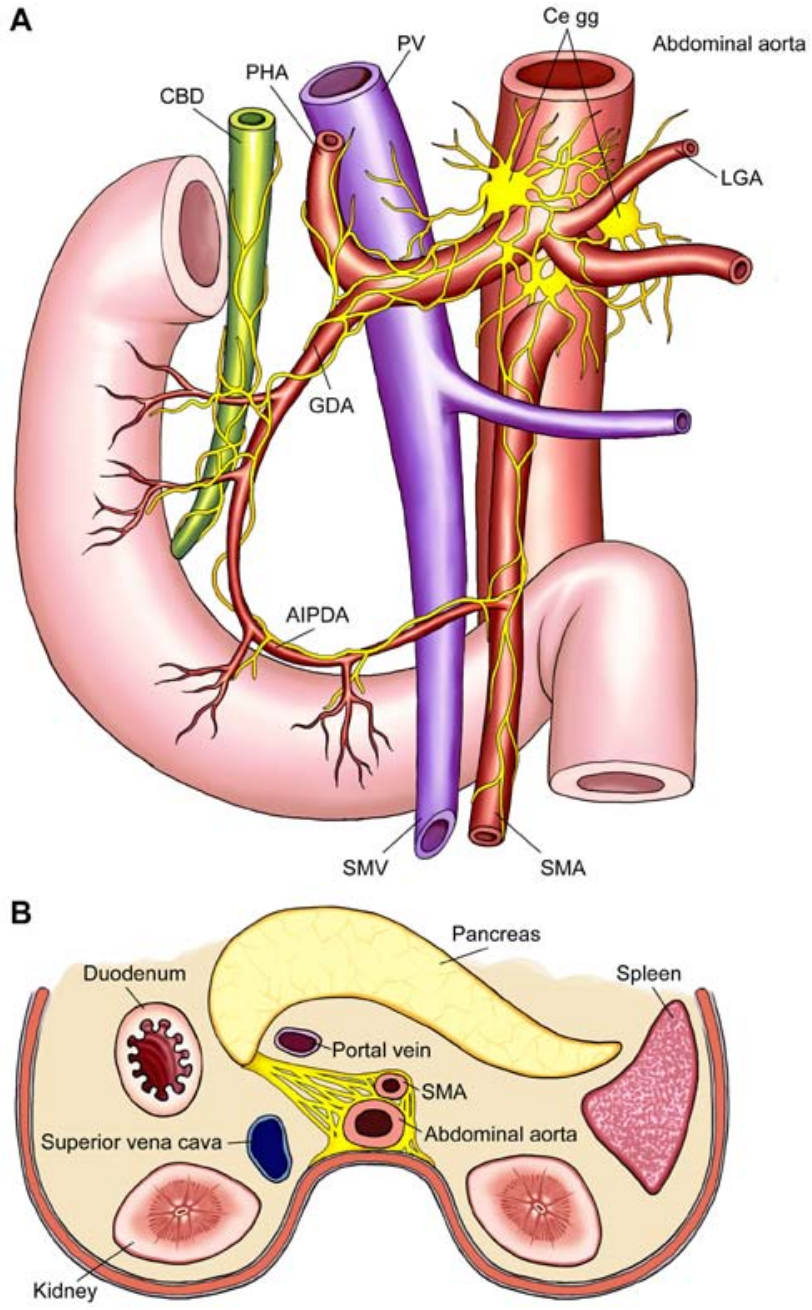

Figure 1. The pancreatic plexus is divided into: i) pancreatic head plexus, which includes the pancreatic head plexus I (PLk ph I) from the right celiac ganglion to the inner upper side of the uncinate process of pancreas and the pancreatic head plexus II (PLk ph II) from the superior mesenteric artery to the inner upper side of the uncinate process of pancreas; ii) the celiac plexus (PL ce); iii) the superior mesenteric artery plexus (PL sma); iv) the hepatoduodenal ligament plexus (PL hdl); v) the hepatic arterial plexus (PL cha); and (vi) the splenic plexus (PL sp).

\section{Anatomy and factors related to neural invasion of pancreatic carcinomas}

The pancreas has a rich nerve supply coming from the internal pancreatic nerve, extrapancreatic nerve and peripancreatic nerve. The internal pancreatic nerve is branched and it travels interlobularly, being accompanied by both the pancreatic vessels and pancreatic duct. Its nerve endings are distributed among the acinar pancreatic cells, creating the necessary infrastructure to meet the requirements of neurotropic growth. The extrapancreatic nerve originates from the right celiac ganglion, goes through the hepatic plexus and the right side of the celiac plexus to form specifically the pancreatic head nerve. A thorough understanding of the classification and distribution of the peripancreatic nerve is critical in pancreatic resection and nerve dissection. During treatment, innervation of the pancreas should be viewed from the perspective of perineural invasion by the pancreatic cancer. A study using post-mortem dissection to observe the nerve fiber distribution in the pancreas and its relationship to tumor invasion was carried out on 9 patients who succumbed to pancreatic cancer (4). The present study found the pancreatic nerve to originate in the region of the superior mesenteric artery. The nerve then runs along the inferior pancreaticoduodenal artery, but not forming large nerve bundles to innervate the pancreatic head. For the pancreatic body and tail, nerve fibers originate from the celiac plexus and go straight into the pancreatic tissues after leaving the celiac plexus, finally branching along the pancreatic duct to form the basis of the anatomical structure of the neural pancreatic plexus (Fig. 1).

\section{Factors associated with neural invasion of pancreatic carcinoma}

Lymph nodes, blood vessels and lymphatic invasion. The relationship between peripancreatic nerve invasion and lymph node metastasis is controversial, but the current thinking is that nerve infiltration is associated with the peripheral lymphatic network structure and nerve distribution (3). Studies have found carcinomas presenting with lymph node metastases are closely related to the incidence of pancreatic nerve invasion. Pancreatic cancer can easily invade the lymphatic system to violate the peripheral nerve. In the superior mesenteric artery (SMA) with peripheral nerve plexus within the reticular lymphatic capillary distribution, pancreatic cancer neural invasion may have a close relationship with lymphangiogenesis (5).

The nature of the tumor. At present, the majority of researchers believe that perineural invasion of pancreatic carcinomas has nothing to do with tumor size and degree of lymphatic invasion, but instead is related to a certain extent to tumor location and its embryologic differentiation (6). In addition, the number of tumor interstitial tissues may also influence the incidence of neural invasion. The interstitial tissues may play an important role in pancreatic cancer neuropathology.

Hyperglycemia. Diabetes is present in $34-40 \%$ of patients with pancreatic cancer, and it has often been diagnosed only in these patients (7). Long-term diabetes is considered to be one of the pathogenic factors of pancreatic cancer, and a recent onset of diabetes may be a manifestation of the cancer (8). With fasting glucose levels, for each additional $0.56 \mathrm{mmol} / 1(10 \mathrm{mg} / \mathrm{dl})$, the corresponding risk of pancreatic cancer increases by roughly $14 \%$ (9). A significant increase in diagnostic frequency of pancreatic cancer has subsequently been associated with a recent diagnosis of diabetes (10). Research has also confirmed that hyperglycemia may be related to neural invasion of pancreatic cancer. In general, cancer cells, high blood sugar, and presence of nerves are three factors that exist in the pancreatic cancer microenvironment; and cancer is the result of interactions among these three factors $(11,12)$.

\section{Molecular biological mechanism of neural invasion in pancreatic carcinomas}

With recent in-depth study of tumor neurobiology, a series of studies have shown that many biological molecules and 


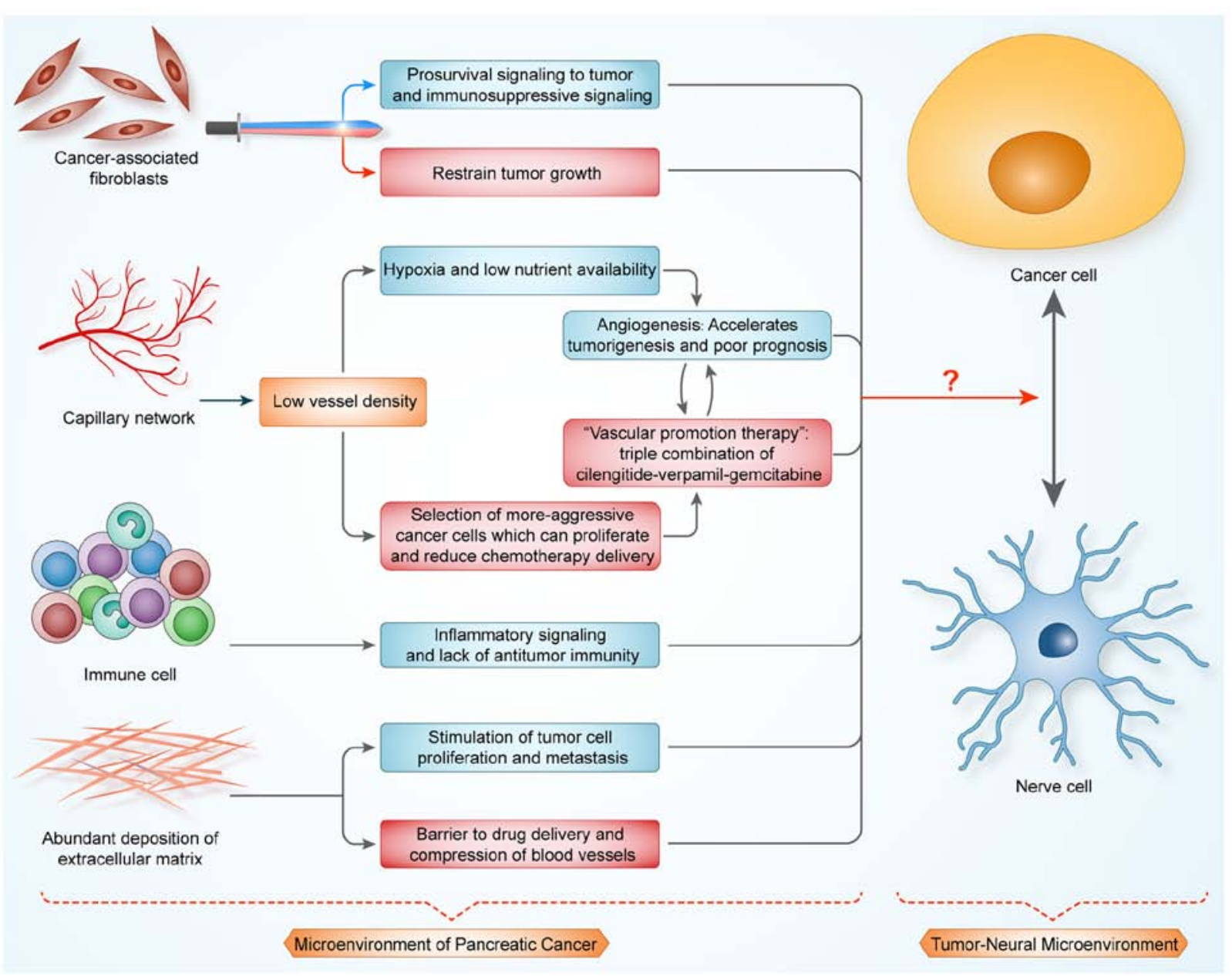

Figure 2. Biologic features of the pancreatic cancer microenvironment.

signaling pathways are closely associated with NI in pancreatic cancer, pointing to a molecular mechanism for NI (3). Relevant pathways include GDNF-RET $(13,14)$, NGF-TrkA, Hedgehog (Hh) $(15,16)$, CX3CR1 stimulation, ATDC (17), vitamin D receptor (18) and matrix metalloproteinases (MMP). The combined effects of NI, secondary tumor masses, destruction of and abnormal regeneration of neurons, and angiogenesis are important factors that signal a poor prognosis in pancreatic cancer.

\section{Composition of pancreatic tumor microenvironment}

The tumor microenvironment is an integrated system composing of tumor cells, stromal cells (e.g. endothelial cells), infiltrating cells (e.g. macrophages and lymphocytes) and the products that they release (19). The tumor microenvironment directly affects the characteristics of NI, and NI changes the microenvironment and the interactions between the tumor and the microenvironment. Therefore, studying the relationship between these factors has important clinical significance (Fig. 2).

Cancer-associated fibroblasts. Pancreatic stellate cells (PSCs) are a type of fibroblast cells that surround the pancreatic lobule and pancreatic acini (20-22). Previous studies have shown that during development of a pancreatic carcinoma, PSCs promote growth and invasion of pancreatic cancer cells which in turn activate PSCs. This constitutes a positive feedback loop that plays an important role in the development of pancreatic carcinomas (23).

The latest study found pancreatic tumor-associated stromal cells to limit tumor development, rather than to promote its development (24). In fact, stromal cells appear to limit further damage through a protective rebuilding mechanism. Even in the end-stages of the disease, the body continues to take measures to try and stall disease progression. However, for pancreatic cancer patients that present with high levels of fibrosis, a combination of gemcitabine and hedgehog inhibitor drugs used to deplete myofibroblasts improves prognosis (25).

Previous treatments have called for complete removal of fibrous tissue, which in all likelihood is not an ideal treatment strategy. New findings may subvert traditional cancer treatment strategies. Our current assumptions about cancer must be re-examined, and only when we can distinguish cells that are directly influenced by growing cancer cells, can we formulate therapeutic strategies to remove cancerous cells with minimal harm. At the same time, we need to make every effort to preserve the beneficial surrounding cells not impacted by tumor to reduce total damage.

Capillary network. Rapid growth of pancreatic tumor tissue requires a rich blood supply. A low density of blood vessels 
places tumor tissue in a hypoxic and nutrient-poor state, which requires angiogenesis to meet its growing needs. Clinical studies show that angiogenesis is related to rapid growth and poor prognosis in patients with pancreatic cancer (26). Angiogenesis depends on a dynamic balance between growth factors and inhibitors $(27,28)$. However, recent studies have found an interesting tumor treatment, called 'vascular promotion therapy' which increases vessel leakage and reduces tumor hypoxia, therefore, suggesting a potentially enhanced intratumoural drug delivery and a reduction in desmoplastic reaction. There is also an interesting approach to cancer treatment by promoting, rather than inhibiting, vascular formation. Treatment with a triple combination of cilengitide-verpamilgemcitabine reduced tumor burden and number of metastases considerably, and these effects were sustained after cessation of treatment (29).

Immune cells. In pancreatic cancer, there is obvious infiltration by immune cells, but these immune cells do not appear to play a role in immune surveillance (30-32). Pancreatic cancer cells evade immune recognition through modification of their surface antigens and changing the surrounding microenvironment. Changes made to the pancreatic tumor microenvironment largely attenuate the immune response to cancerous cells.

Abundant deposition of extracellular matrix. In the pancreatic tumor microenvironment, cancer cells interact with stromal cells (33). During this process, cytokines (34) and products influencing ECM development, such as MMP (35), TGF- $\beta$ (36), HGF (37) and VEGF (38) also play an important role in the formation of the tumor.

\section{Tumor-neural microenvironment}

The existing literature proposes the concept of a tumor-neural microenvironment $(23,39)$ in that cancer cells and nerves constitute a microenvironment which mutually promotes proliferation and inhibits apoptosis. This microenvironment can promote the occurrence of NI, thus making the tumor microenvironment a critical factor in the progression of pancreatic cancer.

In the process of evolution, tumor cells gradually form a favorable microenvironment to foster development, promote tumor cells to grow towards nerve tissue and invade. At the same time, nerve tissue has a specific (favorable) microenvironment to include neurons, glial cells, and their expressed products to exert chemotactic effects on cancer cells, thus promoting invasion. Through NI, the interaction between tumor cells and nerve tissue can further change the microenvironment, thus enhancing NI (3).

As the microenvironment plays an important role in cancer cell invasion and metastasis, changing the microenvironment to reduce the invasiveness of cancer cells has theoretical feasibility. However, since the prognosis is influenced by both tumor and stromal cells, there is not sufficient evidence to show that the microenvironment alone can cause or impair cancer cell invasion. Furthermore, cancer cells exhibit great heterogeneity, such that the 'fittest' cells are selected for survival, allowing the tumor to constantly adapt to changes in the environment. Even when therapeutic strategies successfully intercept the main mechanism of pathogenesis, the problem is often not completely resolved. After cessation of therapy, other (hidden) pathogenesis mechanisms often surface and eventually lead to recurrence. Therefore, the tumor microenvironment is a complex, dynamic network (40), and currently only a few individual factors related to the microenvironment have undergone in depth study. Although the tumor microenvironment plays a complex and important role in cancer pathogenesis, the total influence of the stromal environment is currently poorly understood and necessitates further studies.

\section{Surgical treatment, adjuvant therapy, and monitoring technology to follow neural invasion in pancreatic carcinoma}

The traditional Whipple operation focuses only on excision of the tumor, which often results in inadequate extent of resection and tumor clearance. Lymph node metastasis and pancreatic nerve infiltration are important biological features that occur frequently with pancreatic cancer. The keys to technical improvements on the Whipple operation lie in extending lymph node dissection, performing complete peripancreatic retroperitoneal resection with or without portal vein/mesenteric vascular resection and reconstruction, emphasizing on negative margins, removing all metastatic lymph nodes, preventing peritoneal infiltration, and utilizing microscopic resection techniques to achieve R0 resection (41). Japanese scholars believe that to include the tumor and the surrounding connective tissues, lymphoid tissues, and nervous tissue into a radical resection is the best treatment for pancreatic tumors at present. However, there are serious limitations to such approaches: i) the clinical technology currently available is limited and it is difficult to definitively diagnose the range of neural invasion in the preoperative and intraoperative periods. These predictive measures often rely on lymph node metastasis, tissue grade, tumor size, and other factors that may not accurately characterize the extent of tumor progression; ii) complete resection of retroperitoneal nerve plexus may lead to complications such as severe diarrhea and malnutrition; iii) complete resection involves operating in a deep surgical site with a small visual field and poor exposure in a region surrounded by vital structures. Therefore, there is a balance between the extent of radical resection with the likelihood of recurrence, which is the main dilemma for surgeons. Studies focusing on nerve invasion in pancreatic cancer may help to improve the complete surgical resection rate in early cases, while reducing the postoperative recurrence rate. With a better understanding of the biological characteristics of neurotropic growth in pancreatic cancer, a better decision can be made on the extent of resection and a better long-term post-operative survival can be achieved.

\section{Treatment of retroperitoneal nerve dissection and vascular skeletonization}

Need. Clearing the retroperitoneal nerve plexus, especially the SMA peripheral nerve plexus during surgical treatment of pancreatic cancer has a neuroanatomical basis. 


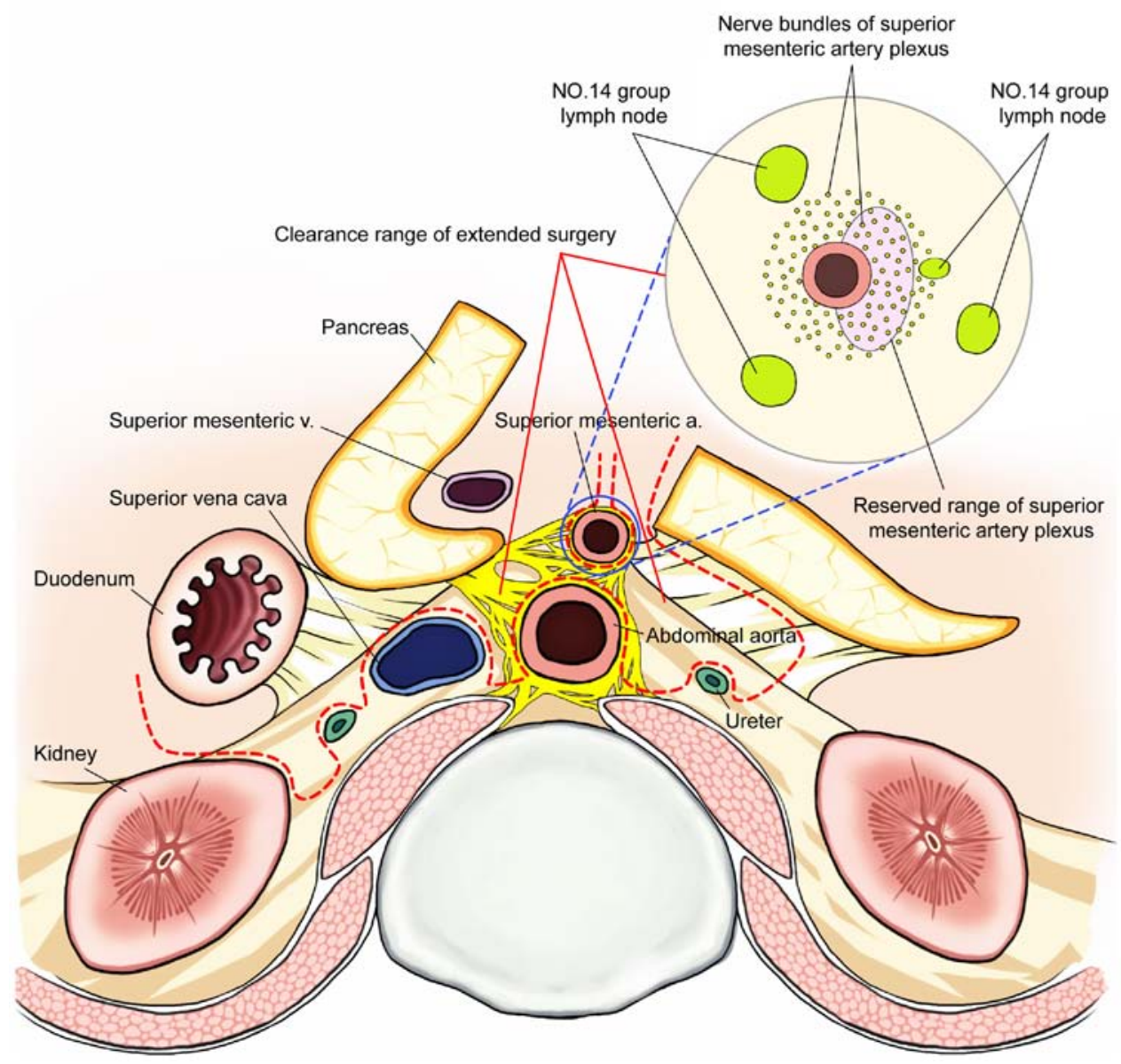

Figure 3. Clearance range of extended surgery (dotted line represents the cutting line) + SMA clearance range profile. 1, upper - lower; Plexus, celiac plexus (left, right celiac ganglion), superior mesenteric artery plexus, plexus around the abdominal aorta, inferior mesenteric artery plexus; Lymph nodes, abdominal aortic hiatus - both sides of the abdominal aorta $\sim 2 \mathrm{~cm}$ below the root of the inferior mesenteric artery; 2, before - after; Lymph node, plexus: root of colon artery - superior mesenteric artery - abdominal aorta: exposure to lumbar artery and psoas muscle level (reserved sympathetic trunk); 3 , right - left; Right renal hilum - the inside of the left ureter.

Recurrences of pancreatic cancer after a Whipple procedure has four patterns: liver metastasis, peritoneal dissemination, retroperitoneal recurrence and distant metastasis. The most important reason for retroperitoneal recurrence to occur is because of perineural invasion. Reports show that $>50 \%$ of pancreatic resection specimens display obvious nerve infiltration. Even after radical pancreaticoduodenectomy (PD), the local retroperitoneal recurrence rate within one year post-surgery is $>80 \%$. The most common site of recurrence is in soft tissues at the rear of the pancreatic head. Therefore, pancreatic nerve invasion has become an independent prognostic marker of long-term survival for pancreatic cancer. The accuracy in prediction is greater than using the $\mathrm{T}$ stage, involved lymph node counts, and other indicators (42). In actual fact, $20-40 \%$ of clinically resectable pancreatic cancers have been pathologically confirmed to be non-radically resected. The main reason is due to the residual margins caused by invasion of peripancreatic plexus. To avoid nerve invasion through other possible means (lymphangiogenesis), the main tumor should be entirely resected (Fig. 3).

What to do?

i) Improvement of existing surgical treatments: intraoperative detection, technology-assisted clearance, left side semicircle clearance around the SMA, or total mesopancreas excision (TMpE)/modified surgical excision.

Auxiliary technology. In 10 patients who died of nondigestive diseases, their corpses were used to show the extent of the NO. 14 group of lymph nodes needed to be dissected to retain the SMA peripheral plexus (43). The results showed 93.7\% of the lymph nodes were located $3 \mathrm{~mm}$ away from the adventitia of the SMA. The average distance between all the SMA lymph nodes from the adventitia was $5.5+2.0 \mathrm{~mm}$. The corresponding distances on the right side and on the left side of the SMA were $5.8 \pm 2.1$ and $5.3 \pm 1.9 \mathrm{~mm}$, respectively. There was no significant difference between the distances on the left and right sides. In addition, the lymph node distributions in the horizontal direction and the vertical direction of SMA were the same. On the other hand, the average width of the nerve plexus wrapping around the SMA (left and right side of the SMA) was $4.2+1.3 \mathrm{~mm}$. Whereas, the positional relationship between the lymph nodes and the nerve plexus in all the 142 lymph nodes which completely wrap around the SMA plexus was $>8$ (5.6\%). The remaining lymph nodes were partially or entirely located outside of the nerve plexus. For the 8 lymph nodes which were embedded in the plexus, 7 (87.5\%) had a distance of $<3 \mathrm{~mm}$ from the SMA arterial adventitia. Combining with the previous data, nearly $94 \%$ of all the NO. 14 group of lymph nodes were 
outside of the SMA peripheral nerve plexus. Thus it was shown that by only retaining the $3-\mathrm{mm}$ thick SMA plexus, almost all of the surrounding lymph nodes can be cleared away, thus, supporting surgical clearance of the NO. 14d group of lymph nodes is technically feasible (43).

Some scholars believe that complete clearance of the connective tissues surrounding the SMA is ideal for patients with infiltrating pancreatic cancer. On taking into account the resulting quality of life, only complete right sided semicircular clearance of the SMA nerve plexus is recommended as these tissues are especially vulnerable to cancer infiltration. As the patient retains the left sided semicircular SMA nerve plexus, even after completing a circumferential resection of the NO. 14 group of lymph nodes, additional intraoperative irradiation may be necessary (43).

There have been reports that show that intraoperative ultrasound or IPUS (intraductal ultrasound) can be used to determine whether the tumor has infiltrated the PV (portal vein) or the pancreatic peripheral nerve plexus intraoperatively. Using IPUS to evaluate invasion of the pancreatic head nerve plexus, the sensitivity, specificity, and accuracy were 94 98 and $97 \%$, respectively (43).

The development of optogenetic technology helps to identify areas to avoid dissecting into. Using optogenetic neuron staining techniques to peroperatively identify the neural location susceptible to injuries, fluorescence can prompt the surgeon to avoid causing accidental injury intraoperatively. As ganglion excision may result in postoperative gastrointestinal dysfunctions and energy absorption disorders, its downstream neurons can be transfected by photosensitive genes and illuminated to preserve gastrointestinal motility and to maximize the patient's post-operative quality of life.

Total mesopancreas excision (TMpE) and modified surgical excision. Research shows that the long-term survival of patients is influenced by the tumor biological characteristics, patient health status, and whether R0 (curative) resection has been achieved (44). Improvements in surgical treatment of pancreatic head carcinomas aim to improve both the surgical resection and the $\mathrm{R} 0$ resection rates. Unfortunately, 20-86\% of patients fail to achieve a real $\mathrm{R} 0$ resection (45-49). In clinical case studies when most operations were considered to be 'radical'/R0 pancreaticoduodenectomy, the positive specimen margin ( $\mathrm{R} 1$ resection) rate with microscopic residual tumor visible under the optical microscope was 30-40\% (47). Although the specific definitions and standards of R1 resection have not yet been internationally standardized, residual cancer $1 \mathrm{~mm}$ from the cutting edge (as visible under a light microscope) is recognized as one of the criteria (50). This explains why many patients with 'R0 resection' do not have long-term survival. For patients with pancreatic head cancer who underwent pancreaticoduodenectomy, the most common site of R1 residual cancer is in the 'mesopancreas' $(51,52)$. Recently, total mesopancreas excision (TMpE) has been implemented and this may help to increase the $\mathrm{R} 0$ resection rate and to improve patient's prognosis (53). Verbeke (50) pointed out that the mesopancreas is the most important part of the retroperitoneal resection margin, and according to this standard, the pancreatic carcinoma $\mathrm{R} 1$ resection rate reached $85 \%$ through improved pathological examination. Therefore, the retroperitoneal margin is the most critical margin related to $\mathrm{R} 0$ resection, and the fact that $\mathrm{R} 1$ resection has been misjudged as $\mathrm{R} 0$ resection in pancreatic head cancers is the main reason why 'R0 resections' have poor results (53).

First described in 2007, the mesopancreas (54) includes the dorsal pancreatic nerve and the lymph tissue layer. Total mesopancreas excision (TMpE) is based on the principle of total mesorectal excision (TME) and includes the peripancreatic lymphatic and adipose tissue layers in an en bloc resection (55). In order to achieve a negative retroperitoneal margin, unlike TME, TMpE does not follow a fixed tissue clearance using complete resection of a predetermined interstitial structure or organ range.

The concept of the mesopancreas triangle was proposed to define the scope of mesopancreas excision (53). The base of the triangle is the superior mesenteric vein and the rear of the portal vein, while the vertex is in the front of the abdominal aorta, between the celiac axis and the starting point of the superior mesenteric artery, to include the superior mesenteric artery and the right side of the semicircle of the celiac plexus. These structures form an inverted triangle (Fig. 4). The range of resection required by $\mathrm{TMpE}$ is partially covered by regional lymph node dissection, but TMpE emphasizes the en bloc resection with soft tissues behind the head of the pancreas to include the lymph nodes and plexus. This is done to complete the surgical field with a three-dimensional (3D) negative edge to achieve a real $\mathrm{R} 0$ resection. According to the pathological examination of intraoperatively-obtained and labeled specimens, the number of cancer positive pancreatic mesopancreas samples in patients with pancreatic head carcinomas was $23 \%$, while the median number of lymph nodes dissected was 24 , and the overall $\mathrm{R} 0$ resection rate was as high as $80 \%$ after TMpE (53).

However, the notion that the pancreatic mesopancreas exists and is involved is not universally accepted at present. Many surgeons believe that there is no fascia surrounding the pancreas and/or that it is not involved in the general or fascia tissue pathology. Therefore, there is no true concept of the pancreatic mesopancreas anatomically (52). However, clinical studies have shown that this structure does exist as a 'category mesopancreas organization' (56). The posterior pancreatic head, superior mesenteric artery, celiac trunk, and the soft tissue around the abdominal aorta margin lack uniform definitions, yet, they comprise the region that is the most common site of invasion and metastasis in pancreatic head carcinomas. The objective facts that residual tumors and local recurrence often occur in this area after surgical resection help to define this area as the pancreatic mesopancreas, even though there may not be any enveloping fascia. Clearer definitions and recognition of this area can help to standardize the rate of radical cure of pancreatic head cancer and improve efficacy. The principles of TMpE emphasize that pancreatic head carcinoma resection should be combined with en bloc of this area to achieve R0 resection. When compared with other current surgical methods through existing clinical studies, TMpE shows no significant differences in operation time, intraoperative blood loss, incidence of complications, postoperative hospital stay or perioperative mortality. Therefore, despite all the controversies, TMpE is a comparatively safe and viable option for treatment of pancreatic head tumors (53). 


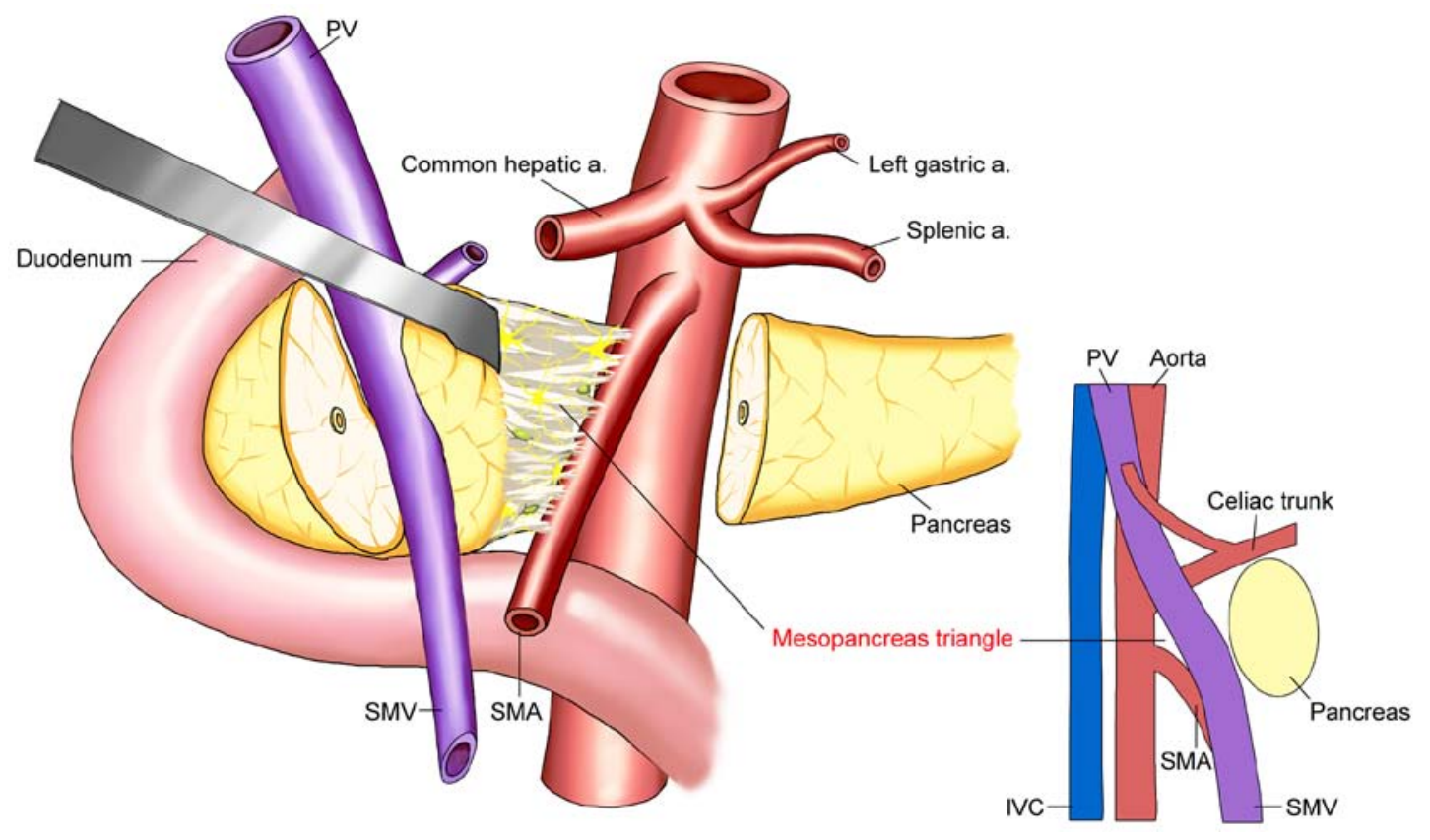

Figure 4. Mesopancreas triangle which needs to be cleared for pancreatic head carcinoma for better R0 resection.

Viewed from the perspective of embryology, due to a duodenal loop, the 'meso' disappears during embryonic development (57). The embryonic mesentery facilitates the pancreatic germ attachment to the abdominal wall, thus, forming the pancreatic mesopancreas. This is why the pancreatic mesopancreas must be completely resected up to the SMA. The concept of 'meso-pancreatoduodenum excision' (tMPDe) was put forward to address this (58). The meso-pancreatoduodenum is defined as a mesangial mesentery supplied by the inferior pancreaticoduodenal artery (IPDA), which runs towards the third and fourth portions of the duodenum and the proximal jejunal mesentery supplied by the first jejunal artery (FJA) at the back of the SMA. During tMPDe, the scope of the TmpE (Fig. 5) is extended to the left side of the SMA from the coronal plane to ensure thorough cleaning of the retroperitoneal margin. As a result the $\mathrm{R} 0$ resection rate significantly increases when compared with the standard pancreaticoduodenectomy procedure (93 vs. 60\%) (58).

However, current TMpE studies are retrospective studies. Larger cohort studies and randomized controlled trials are needed to evaluate the clinical effects. Also, the long-term prognosis of these patients remains unclear. Some clinical studies using extended retroperitoneal clearance failed to show that extended resection improves patient prognosis. Although the concepts of mesopancreas and $\mathrm{TMpE}$ are controversial, there are some other reports which show TMpE has definite advantages. We believe TMpE is an entirely new concept that requires further evaluation. Its clinical application may improve the prognosis of patients with pancreatic carcinomas, but large randomized clinical studies are necessary to demonstrate its true role in the treatment of pancreatic head cancer.

ii) Breakthroughs in the existing operation procedures using a circumferential resection plus adjuvant therapy? Extensive resection of the retroperitoneal nerve plexus will cause the bowel to lose its dual innervation, thus, resulting in serious complications such as severe diarrhea and malabsorption of nutrients. These can be difficult to control even with medication (59) and can significantly impair the quality of life after operation. In some situations, these complications can be life-threatening. However, in clinical practice, the incidence of postoperative intractable diarrhea does not occur more frequently than after extensive resection, suggesting that the incidence may be low and/or readily controlled (58). However, if the tumor is circumferentially wrapping around or is invading the SMA, radical operations should not be attempted. In this case, palliative operation or implantation of $\mathrm{I}^{125}$ are better options. If preoperative imaging and intraoperative exploration only detect suspicious lymph node metastasis on the left edge of the SMA, the SMA can theoretically be completely skeletonized, which is then combined with optogenetic adjuvant therapy.

Based on the concept of TMpE (en bloc resection and R0 resection), the 'no touch' tumor principle and integrated with a large number of clinical reports, the SMA should be dissected first to determine whether it is feasible to perform radical resection. Total mesopancreas excision should be carried out to remove the lymphatics and blood vessels in the mesopancreas, which falls in line with the principle of 'no contact' tumor resection before the pancreaticoduodenectomy (58).

\section{What do we need?}

i) Randomized controlled studies worldwide at this stage do not support extended resection and extended lymph node clearance. The latest clinical study on 200 patients with pancreatic head carcinomas (59) showed no improvement in long-term survival by using extended resection, including extended lymph node dissection and plexus dissection when compared to the traditional standard pancreaticoduodenectomy (Whipple procedure). This large prospective study showed that extended resection did not improve long-term outcomes. 


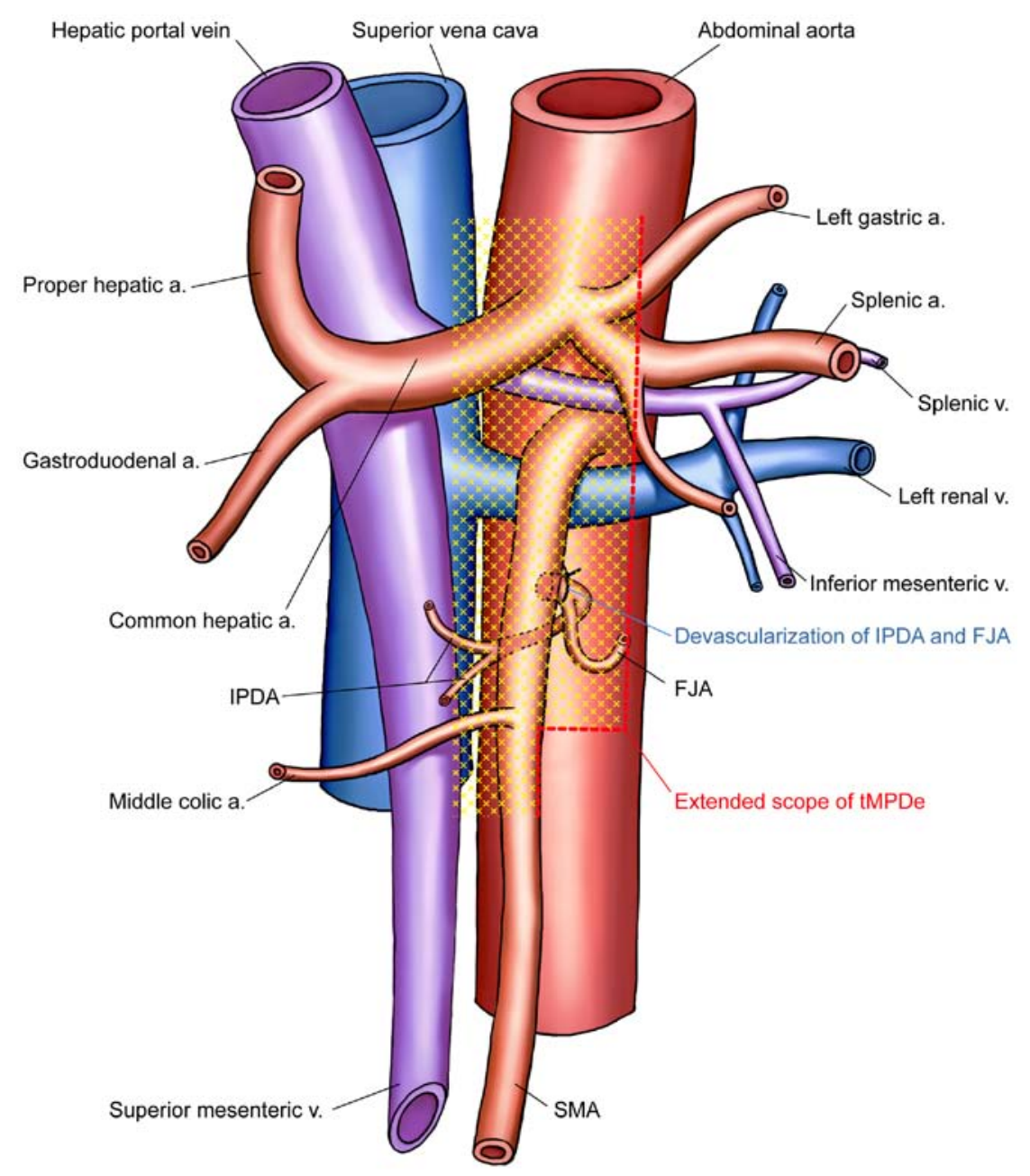

Figure 5. The key extent of meso-pancreatoduodenum excision (tMPDe): i) The left side semicircle lymphadenectomy around the SMA from the origin of the MCA up to the origin of the SMA in a longitudinal direction on the ventral side of the SMA. The origin of the common trunk of the inferior pancreaticoduodenal artery (IPDA) and first jejunal artery (FJA) need to be secured at the left posterior side of the SMA. A right-side semicircle lymphadenectomy is then performed, soft connective tissue around the SMA containing lymph nodes, mesopancreas, and PL around the SMV is dissected up to the origin of the SMA in a longitudinal direction. After completion of the circumferential lymphadenectomy, the IPDA and FJA are ligated and divided. ii) If the portal venous system was involved in tumor invasion, a quick resection of the vein followed by an end-to-end anastomosis was conducted. The resected specimens demonstrated that the mesoduodenum, which is fed by the IPDA, and the jejunal mesentery, which is dominated by the FJA, form a common mesentery, and thus named this common mesentery the 'meso-pancreatoduodenum'.

However, the present review has many debatable points. For example, in the cases of radical operation, it is not clear what criteria were used to define the extent of clearance or how the SMA peripheral nerve plexus was distinguished from the No. 14d group of lymph node during dissection. The review also mentioned on several occasions that complete circumferential clearance of the SMA plexus would inevitably lead to intractable diarrhea, poor nutrient intake, and immune dysfunction, which would affect the postoperative quality of life and survival of the patients. Therefore, in the extended resection group there was no clearance of the left half of the SMA. However, there were no clear data in the medical literature to indicate the severity and intractability of the diarrhea. References used were at least 10 years old. The lengths of hospital stay mainly caused by the national health care system were also different between the two groups, which affected the accuracy of the results. Notably, the report also indicated that in the extended resection group there were significantly more patients with peritoneal metastasis.
The report also noted that in the standard group and the extended group, operation time and blood loss were significantly different. As dissection was performed near to major blood vessels and nerves in the extended group, balancing the risk of prolonged operation times, increased bleeding volumes, and surgical complications with the potential extension of postoperative survival warrants further investigations.

Retrospective clinical data from Japan indicated that extended resection could result in significantly improved survival $(60,61)$. However, randomized controlled trials in Europe and the United States do not support the use of extended resection in pancreatic cancer (61). Prospective randomized studies in Europe and the United States showed that the survival rate was not significantly different between extended radical and standard operations, while the extended radical operation had a higher rate of surgical complications (62).

In summary, each center has its own view on the scope and extent of retroperitoneal dissection. Most European and American scholars tend to advocate for a standard Whipple 
procedure. For extended retroperitoneal dissection, information from the Japanese scholars cannot lead to a conclusion as to whether an extended resection is superior to a standard Whipple in long-term survival outcomes.

ii) Costs related to pancreatic cancer management. The majority of patients with pancreatic cancer are diagnosed late. The patients are old, and the survival rate is very low because of rapid tumor progression (63). In 2014, the median age of patients diagnosed with pancreatic cancer was 71-years old in the United States. The incidence has increased each year, and the numbers of new cases and fatal cases are expected to reach 46,420 and 39,590, respectively. Pancreatic cancer is ranked number four in malignant tumor mortality, and the 5-year survival rate is $<6 \%(64,65)$. The main cost of diagnosis and treatment for these patients include costs for hospitalization, postoperative drug treatment, nursing and other costs $(66,67)$. Pancreatic resection is arguably the most complex and difficult surgery in the medical profession. Even eliminating all objective conditions, the technical level of the surgeons and other human factors, the risk of surgical complications remains high. Surgical complications (aside from the cancer) can be life-threatening. At the same time, with increasing costs for long-term postoperative care after reconstruction of digestive tract and the risk of resulting malabsorption (68), the costs to the patient's family, patient and society are a heavy burden that will increase with disease incidence.

\section{Optogenetic technology with pancreatic cancer for detection and adjuvant therapy}

To study the basis of pancreatic neural invasion which includes the mechanisms of neural invasion, optogenetic technology can be used to control the optics of a cell after making it to express a light-sensitive protein with genetic techniques which integrates optics, electrophysiology, genetic engineering and other disciplines. By using light regulation of specific neuronal cell types, the function of neural circuits and the specific control of biological behavior can be studied $(69,70)$. Optogenetic technology is being applied more widely because of its ease in handling, intact stimulus, high temporal and spatial precision, quantifiability and repeatability $(71,72)$. The core of optogenetic technology includes real-time imaging technology of specific neurons and light-sensitive control technology. As a possible way to go beyond traditional neuroscience research methods and to regulate neuronal activity, channelrhodopsin-2 (ChR2), a light-sensitive ion channel protein that can cause changes in membrane potential in response to light, represents a useful tool $(73,74)$. Optogenetic technology can target ChR2 to specific neural circuits and control the function effectively. From the neural networks of the nematode to brain research in rhesus monkeys, ChR2 has been systemically tested in higher level animals (75). From basic research studies on neural circuits to clinical diseases of the nervous system, ChR2 has become a new method to explore neural system diseases. With a more practical significance, optogenetic technology can be applied in vivo (76). Some scholars use this technique (with good results) in conjunction with lentivirus application to alter the pathogenesis of sleep disorders (77).
During pancreatic resection, injured nerves can cause postoperative gastrointestinal disorders. However, injury is common due to the limited resolution of the naked eye, making it difficult to visualize tiny nerve endings. Real-time neuronal staining using optogenetic technology in vivo allows for the visualization of such nerves and their distribution to avoid accidental injury. Unilateral or bilateral celiac ganglia that have been violated by pancreatic cancer cells must be removed. Considering that gastrointestinal disorders can significantly reduce the quality of life, downstream neurons can be transfected with light-sensitive proteins and illuminated to preserve the nerves and thus gastrointestinal motility.

ChR2 can also be used to regulate other cellular calcium signaling regulatory pathways (78), such as the calcium signaling pathway to control pancreatic stellate cells, which is helpful to further understand how they affect neurons with regards to support, nourish, protect and communicate. Such techniques can be used to further explore the molecular mechanisms of neural invasion in pancreatic carcinomas $(79,80)$.

With development of new technology and identification of light-sensitive channel proteins with long-term stability which can then be expressed in specific neurons and monitored using fast 3D beam scanning methods, we have reasons to believe that pancreatic cancers will soon be treatable using optogenetic technology (81).

\section{Conclusions}

In conclusion, a better understanding of the mechanisms which are involved in NI and the role of NI in pancreatic cancer progression is essential. Promoting advanced preoperative evaluation systems, strengthening the collaboration of multi-disciplinary teams (MDT), making a reasonable choice of treatment, and continuing to standardize pancreatic surgical procedures are the keys to achieving higher $\mathrm{R} 0$ resection rates and improving prognoses of patients with pancreatic cancer.

\section{Acknowledgements}

The present study was supported by the Funds of the National Natural Science Foundation of China (61371066), the Key Medical Talents of Jiangsu Province (RC2011090), and the 333 Program for High Level Talents of Jiangsu Province (grant no. 2011III-2640).

\section{References}

1. Liebig C, Ayala G, Wilks JA, Berger DH and Albo D: Perineural invasion in cancer: A review of the literature. Cancer 115: 3379-3391, 2009.

2. Alderton GK: Microenvironment: An exercise in restraint. Nat Rev Cancer 14: 449, 2014.

3. Bapat AA, Hostetter G, Von Hoff DD and Han H: Perineural invasion and associated pain in pancreatic cancer. Nat Rev Cancer 11: 695-707, 2011.

4. Yi SQ, Miwa K, Ohta T, Kayahara M, Kitagawa H, Tanaka A, Shimokawa T, Akita K and Tanaka S: Innervation of the pancreas from the perspective of perineural invasion of pancreatic cancer. Pancreas 27: 225-229, 2003.

5. Cheng P, Jin G, Hu X, Shi M, Zhang Y, Liu R, Zhou Y, Shao C, Zheng $\mathrm{J}$ and Zhu M: Analysis of tumor-induced lymphangiogenesis and lymphatic vessel invasion of pancreatic carcinoma in the peripheral nerve plexus. Cancer Sci 103: 1756-1763, 2012. 
6. Makino I, Kitagawa H, Ohta T, Nakagawara H, Tajima H, Ohnishi I, Takamura H, Tani T and Kayahara M: Nerve plexus invasion in pancreatic cancer: Spread patterns on histopathologic and embryological analyses. Pancreas 37: 358-365, 2008.

7. Chari ST, Leibson CL, Rabe KG, Timmons LJ, Ransom J, de Andrade M and Petersen GM: Pancreatic cancer-associated diabetes mellitus: Prevalence and temporal association with diagnosis of cancer. Gastroenterology 134: 95-101, 2008.

8. Satija A, Spiegelman D, Giovannucci E and Hu FB: Type 2 diabetes and risk of cancer. BMJ 350: g7707, 2015.

9. Liao WC, Tu YK, Wu MS, Lin JT, Wang HP and Chien KL: Blood glucose concentration and risk of pancreatic cancer: Systematic review and dose-response meta-analysis. BMJ 349: g7371, 2015.

10. Pannala R, Basu A, Petersen GM and Chari ST: New-onset diabetes: A potential clue to the early diagnosis of pancreatic cancer. Lancet Oncol 10: 88-95, 2009.

11. Giovannucci E and Michaud D: The role of obesity and related metabolic disturbances in cancers of the colon, prostate, and pancreas. Gastroenterology 132: 2208-2225, 2007.

12. Li J and Ma Q: Hyperglycemia promotes the perineural invasion in pancreatic cancer. Med Hypotheses 71: 386-389, 2008.

13. He S, Chen CH, Chernichenko N, He S, Bakst RL, Barajas F, Deborde S, Allen PJ, Vakiani E, Yu Z, et al: GFR $\alpha 1$ released by nerves enhances cancer cell perineural invasion through GDNF-RET signaling. Proc Natl Acad Sci USA 111 E2008-E2017, 2014

14. Gao L, Bo H, Wang Y, Zhang J and Zhu M: Neurotrophic factor artemin promotes invasiveness and neurotrophic function of pancreatic adenocarcinoma in vivo and in vitro. Pancreas 44 134-143, 2015

15. Martínez-Bosch N, Fernández-Barrena MG, Moreno M, OrtizZapater E, Munné-Collado J, Iglesias M, André S, Gabius HJ, Hwang RF, Poirier F, et al: Galectin-1 drives pancreatic carcinogenesis through stroma remodeling and Hedgehog signaling activation. Cancer Res 74: 3512-3524, 2014

16. Li X, Wang Z, Ma Q, Xu Q, Liu H, Duan W, Lei J, Ma J, Wang X, Lv S, et al: Sonic hedgehog paracrine signaling activates stromal cells to promote perineural invasion in pancreatic cancer. Clin Cancer Res 20: 4326-4338, 2014.

17. Wang L, Yang H, Abel EV, Ney GM, Palmbos PL, Bednar F, Zhang Y, Leflein J, Waghray M, Owens S, et al: ATDC induces an invasive switch in KRAS-induced pancreatic tumorigenesis. Genes Dev 29: 171-183, 2015.

18. Sherman MH, Yu RT, Engle DD, Ding N, Atkins AR, Tiriac H, Collisson EA, Connor F, Van Dyke T, Kozlov S, et al: Vitamin $\mathrm{D}$ receptor-mediated stromal reprogramming suppresses pancreatitis and enhances pancreatic cancer therapy. Cell 159: 80-93, 2014.

19. Witz IP and Levy-Nissenbaum O: The tumor microenvironment in the post-PAGET era. Cancer Lett 242: 1-10, 2006.

20. Helm O, Mennrich R, Petrick D, Goebel L, Freitag-Wolf S, Röder C, Kalthoff H, Röcken C, Sipos B, Kabelitz D, et al Comparative characterization of stroma cells and ductal epithelium in chronic pancreatitis and pancreatic ductal adenocarcinoma. PLoS One 9: e94357, 2014.

21. Hidalgo M: Pancreatic cancer. N Engl J Med 362: 1605-1617, 2010.

22. Gore $\mathrm{J}$ and Korc M: Pancreatic cancer stroma: Friend or foe? Cancer Cell 25: 711-712, 2014.

23. Heinemann V, Reni M, Ychou M, Richel DJ, Macarulla T and Ducreux M: Tumour-stroma interactions in pancreatic ductal adenocarcinoma: Rationale and current evidence for new therapeutic strategies. Cancer Treat Rev 40: 118-128, 2014.

24. Rhim AD, Oberstein PE, Thomas DH, Mirek ET, Palermo CF, Sastra SA, Dekleva EN, Saunders T, Becerra CP, Tattersall IW, et al: Stromal elements act to restrain, rather than support, pancreatic ductal adenocarcinoma. Cancer Cell 25: 735-747, 2014.

25. Özdemir BC, Pentcheva-Hoang T, Carstens JL, Zheng X, Wu CC, Simpson TR, Laklai H, Sugimoto H, Kahlert C, Novitskiy SV, et al: Depletion of carcinoma-associated fibroblasts and fibrosis induces immunosuppression and accelerates pancreas cancer with reduced survival. Cancer Cell 25: 719-734, 2014.

26. Karademir S, Sökmen S, Terzi C, Sağol O, Özer E, Astarcioğlu H, Coker A and Astarcioğlu I: Tumor angiogenesis as a prognostic predictor in pancreatic cancer. J Hepatobiliary Pancreat Surg 7: $489-495,2000$
27. Khorana AA, Ahrendt SA, Ryan CK, Francis CW, Hruban RH, $\mathrm{Hu}$ YC, Hostetter G, Harvey J and Taubman MB: Tissue factor expression, angiogenesis, and thrombosis in pancreatic cancer. Clin Cancer Res 13: 2870-2875, 2007.

28. Wente MN, Keane MP, Burdick MD, Friess H, Büchler MW, Ceyhan GO, Reber HA, Strieter RM and Hines OJ: Blockade of the chemokine receptor CXCR2 inhibits pancreatic cancer cellinduced angiogenesis. Cancer Lett 241: 221-227, 2006.

29. Wong PP, Demircioglu F, Ghazaly E, Alrawashdeh W, Stratford MR, Scudamore CL, Cereser B, Crnogorac-Jurcevic T, McDonald S, Elia G, et al: Dual-action combination therapy enhances angiogenesis while reducing tumor growth and spread. Cancer Cell 27: 123-137, 2015.

30. Clark CE, Hingorani SR, Mick R, Combs C, Tuveson DA and Vonderheide RH: Dynamics of the immune reaction to pancreatic cancer from inception to invasion. Cancer Res 67: 9518-9527, 2007.

31. Bayne LJ, Beatty GL, Jhala N, Clark CE, Rhim AD, Stanger BZ and Vonderheide RH: Tumor-derived granulocyte-macrophage colony-stimulating factor regulates myeloid inflammation and T cell immunity in pancreatic cancer. Cancer Cell 21: 822-835, 2012.

32. Greten TF: Myeloid-derived suppressor cells in pancreatic cancer: more than a hidden barrier for antitumour immunity? Gut 63: 1690-1691, 2014.

33. Neesse A, Michl P, Frese KK, Feig C, Cook N, Jacobetz MA, Lolkema MP, Buchholz M, Olive KP, Gress TM, et al: Stromal biology and therapy in pancreatic cancer. Gut 60: 861-868, 2011.

34. Schlomann U, Koller G, Conrad C, Ferdous T, Golfi P, Garcia AM, Höfling S, Parsons M, Costa P, Soper R, et al: ADAM8 as a drug target in pancreatic cancer. Nat Commun 6: 6175, 2015.

35. Kahlert C, Fiala M, Musso G, Halama N, Keim S, Mazzone M, Lasitschka F, Pecqueux M, Klupp F, Schmidt T, et al: Prognostic impact of a compartment-specific angiogenic marker profile in patients with pancreatic cancer. Oncotarget 5: 12978-12989, 2014.

36. Jacobs EJ, Newton CC, Silverman DT, Nogueira LM, Albanes D, Männistö S, Pollak M and Stolzenberg-Solomon RZ: Serum transforming growth factor- $\beta 1$ and risk of pancreatic cancer in three prospective cohort studies. Cancer Causes Control 25: 1083-1091, 2014.

37. Hermann PC, Huber SL, Herrler T, Aicher A, Ellwart JW, Guba M, Bruns CJ and Heeschen C: Distinct populations of cancer stem cells determine tumor growth and metastatic activity in human pancreatic cancer. Cell Stem Cell 1: 313-323, 2007.

38. Bai X, Zhi X, Zhang Q, Liang F, Chen W, Liang C, Hu Q, Sun X, Zhuang $\mathrm{Z}$ and Liang $\mathrm{T}$ : Inhibition of protein phosphatase $2 \mathrm{~A}$ sensitizes pancreatic cancer to chemotherapy by increasing drug perfusion via HIF-1 $\alpha$-VEGF mediated angiogenesis. Cancer Lett 355: 281-287, 2014.

39. Farrow B, Albo D and Berger DH: The role of the tumor microenvironment in the progression of pancreatic cancer. J Surg Res 149: 319-328, 2008.

40. Ryan DP, Hong TS and Bardeesy N: Pancreatic adenocarcinoma. N Engl J Med 371: 1039-1049, 2014.

41. Bockhorn M, Uzunoglu FG, Adham M, Imrie C, Milicevic M, Sandberg AA, Asbun HJ, Bassi C, Büchler M, Charnley RM, et al; International Study Group of Pancreatic Surgery: Borderline resectable pancreatic cancer: A consensus statement by the International Study Group of Pancreatic Surgery (ISGPS). Surgery 155: 977-988, 2014.

42. Fouquet T, Germain A, Brunaud L, Bresler L and Ayav A: Is perineural invasion more accurate than other factors to predict early recurrence after pancreatoduodenectomy for pancreatic head adenocarcinoma? World J Surg 38: 2132-2137, 2014.

43. Kimura W and Makuuchi M: Suihi geka no youten to mouten. Bunkodo, Tokyo, 2009 (In Japanese).

44. Wagner M, Redaelli C, Lietz M, Seiler CA, Friess H and Büchler MW: Curative resection is the single most important factor determining outcome in patients with pancreatic adenocarcinoma. Br J Surg 91: 586-594, 2004.

45. Weitz J, Rahbari N, Koch M and Büchler MW: The 'artery first' approach for resection of pancreatic head cancer. J Am Coll Surg 210: e1-e4, 2010

46. Verbeke CS, Leitch D, Menon KV, McMahon MJ, Guillou PJ and Anthoney A: Redefining the R1 resection in pancreatic cancer. Br J Surg 93: 1232-1237, 2006. 
47. Esposito I, Kleeff J, Bergmann F, Reiser C, Herpel E, Friess H, Schirmacher P and Büchler MW: Most pancreatic cancer resections are R1 resections. Ann Surg Oncol 15: 1651-1660, 2008.

48. Cameron JL, Riall TS, Coleman J and Belcher KA: One thousand consecutive pancreaticoduodenectomies. Ann Surg 244: 10-15, 2006.

49. Schmidt CM, Powell ES, Yiannoutsos CT, Howard TJ, Wiebke EA, Wiesenauer CA, Baumgardner JA, Cummings OW, Jacobson LE, Broadie TA, et al: Pancreaticoduodenectomy: A 20-year experience in 516 patients. Arch Surg 139: 718-725, discussion 725-727, 2004.

50. Verbeke CS: Resection margins and R1 rates in pancreatic cancer - are we there yet? Histopathology 52: 787-796, 2008.

51. Gaedcke J, Gunawan B, Grade M, Szöke R, Liersch T, Becker H and Ghadimi BM: The mesopancreas is the primary site for R1 resection in pancreatic head cancer: Relevance for clinical trials. Langenbecks Arch Surg 395: 451-458, 2010.

52. Agrawal MK, Thakur DS, Somashekar U, Chandrakar SK and Sharma D: Mesopancreas: Myth or reality? JOP 11: 230-233, 2010.

53. Adham M and Singhirunnusorn J: Surgical technique and results of total mesopancreas excision (TMpE) in pancreatic tumors. Eur J Surg Oncol 38: 340-345, 2012.

54. Gockel I, Domeyer M, Wolloscheck T, Konerding MA and Junginger T: Resection of the mesopancreas (RMP): A new surgical classification of a known anatomical space. World J Surg Oncol 5: 44, 2007.

55. Bouassida M, Mighri MM, Chtourou MF, Sassi S, Touinsi H, Hajji H and Sassi S: Retroportal lamina or mesopancreas? Lessons learned by anatomical and histological study of thirty three cadaveric dissections. Int J Surg 11: 834-836, 2013.

56. Dumitrascu T and Popescu I: Total mesopancreas excision in pancreatic head adenocarcinoma: The same impact as total mesorectal excision in rectal carcinoma? Comment on article "surgical technique and results of total mesopancreas excision in pancreatic tumours" by Adham M and Singhirunnusorn J, Eur J Surg Oncol, 2012. Eur J Surg Oncol 38: 725, author reply 726, 2012.

57. Chowdappa $\mathrm{R}$ and Challa VR: Mesopancreas in pancreatic cancer: where do we stand - review of literature. Indian J Surg Oncol 6: 69-74, 2014.

58. Kawabata Y, Tanaka T, Nishi T, Monma H, Yano S and Tajima Y: Appraisal of a total meso-pancreatoduodenum excision with pancreaticoduodenectomy for pancreatic head carcinoma. Eur J Surg Oncol 38: 574-579, 2012.

59. Jang JY, Kang MJ, Heo JS, Choi SH, Choi DW, Park SJ, Han SS, Yoon DS, Yu HC, Kang KJ, et al: A prospective randomized controlled study comparing outcomes of standard resection and extended resection, including dissection of the nerve plexus and various lymph nodes, in patients with pancreatic head cancer. Ann Surg 259: 656-664, 2014.

60. Nimura Y, Nagino M, Takao S, Takada T, Miyazaki K, Kawarada Y, Miyagawa S, Yamaguchi A, Ishiyama S, Takeda Y, et al: Standard versus extended lymphadenectomy in radical pancreatoduodenectomy for ductal adenocarcinoma of the head of the pancreas: Long-term results of a Japanese multicenter randomized controlled trial. J Hepatobiliary Pancreat Sci 19: 230-241, 2012.

61. Michalski CW, Kleeff J, Wente MN, Diener MK, Büchler MW and Friess H: Systematic review and meta-analysis of standard and extended lymphadenectomy in pancreaticoduodenectomy for pancreatic cancer. Br J Surg 94: 265-273, 2007.

62. Farnell MB, Pearson RK, Sarr MG, DiMagno EP, Burgart LJ, Dahl TR, Foster N and Sargent DJ; Pancreas Cancer Working Group: A prospective randomized trial comparing standard pancreatoduodenectomy with pancreatoduodenectomy with extended lymphadenectomy in resectable pancreatic head adenocarcinoma. Surgery 138: 618-628, discussion 628-630, 2005.
63. Lee A, Chiu CH, Cho MWA, Gomersall CD, Lee KF, Cheung YS and Lai PB: Factors associated with failure of enhanced recovery protocol in patients undergoing major hepatobiliary and pancreatic surgery: A retrospective cohort study. BMJ Open 4: e005330, 2014.

64. Siegel R, Ma J, Zou Z and Jemal A: Cancer statistics, 2014. CA Cancer J Clin 64: 9-29, 2014.

65. DeSantis CE, Lin CC, Mariotto AB, Siegel RL, Stein KD, Kramer JL, Alteri R, Robbins AS and Jemal A: Cancer treatment and survivorship statistics, 2014. CA Cancer J Clin 64: 252-271, 2014.

66. Pfister DG: The just price of cancer drugs and the growing cost of cancer care: Oncologists need to be part of the solution. J Clin Oncol 31: 3487-3489, 2013

67. Short MN, Aloia TA and Ho V: The influence of complications on the costs of complex cancer surgery. Cancer 120: 1035-1041, 2014.

68. Sanford DE, Sanford AM, Fields RC, Hawkins WG Strasberg SM and Linehan DC: Severe nutritional risk predicts decreased long-term survival in geriatric patients undergoing pancreaticoduodenectomy for benign disease. J Am Coll Surg 219: 1149-1156, 2014.

69. Deisseroth K, Feng G, Majewska AK, Miesenböck G, Ting A and Schnitzer MJ: Next-generation optical technologies for illuminating genetically targeted brain circuits. J Neurosci 26: 10380-10386, 2006.

70. Fenno L, Yizhar O and Deisseroth K: The development and application of optogenetics. Annu Rev Neurosci 34: 389-412, 2011.

71. Nagel G, Brauner M, Liewald JF, Adeishvili N, Bamberg E and Gottschalk A: Light activation of channelrhodopsin-2 in excitable cells of Caenorhabditis elegans triggers rapid behavioral responses. Curr Biol 15: 2279-2284, 2005.

72. Li X, Gutierrez DV, Hanson MG, Han J, Mark MD, Chiel H, Hegemann P, Landmesser LT and Herlitze S: Fast noninvasive activation and inhibition of neural and network activity by vertebrate rhodopsin and green algae channelrhodopsin. Proc Natl Acad Sci USA 102: 17816-17821, 2005.

73. Zhang F, Wang LP, Boyden ES and Deisseroth K: Channelrhodopsin-2 and optical control of excitable cells. Nat Methods 3: 785-792, 2006

74. Cardin JA, Carlén M, Meletis K, Knoblich U, Zhang F, Deisseroth K, Tsai LH and Moore CI: Targeted optogenetic stimulation and recording of neurons in vivo using cell-typespecific expression of Channelrhodopsin-2. Nat Protoc 5: 247-254, 2010

75. Han X, Qian X, Bernstein JG, Zhou HH, Franzesi GT, Stern P, Bronson RT, Graybiel AM, Desimone R and Boyden ES: Millisecond-timescale optical control of neural dynamics in the nonhuman primate brain. Neuron 62: 191-198, 2009.

76. Yizhar O, Fenno LE, Davidson TJ, Mogri M and Deisseroth K: Optogenetics in neural systems. Neuron 71: 9-34, 2011.

77. Adamantidis AR, Zhang F, Aravanis AM, Deisseroth K and de Lecea L: Neural substrates of awakening probed with optogenetic control of hypocretin neurons. Nature 450: 420-424, 2007.

78. Hung $\mathrm{J}$ and Colicos MA: Astrocytic $\mathrm{Ca}^{2+}$ waves guide CNS growth cones to remote regions of neuronal activity. PLoS One 3: e3692, 2008

79. Adelsberger H, Grienberger C, Stroh A and Konnerth A: In vivo calcium recordings and channelrhodopsin-2 activation through an optical fiber. Cold Spring Harbor Protocols 2014: pdb. prot084145, 2014.

80. Zhang Y, Yue J, Ai M, Ji Z, Liu Z, Cao X and Li L: Channelrhodopsin-2-expressed dorsal root ganglion neurons activates calcium channel currents and increases action potential in spinal cord. Spine 39: E865-E869, 2014.

81. Fenno LE and Deisseroth K: Optogenetic tools for control of neural activity. Optical Imaging of Neocortical Dynamics. Springer, pp73-86, 2014. 